\title{
CARACTERIZACIÓN DE NIÑOS CON VIH POR TRANSMISIÓN MATERNO-INFANTIL ATENDIDOS EN HOSPITALES DE LIMA, PERÚ
}

\author{
Carlos Velásquez-Vásquez(1),a,b, Marcos Espinola-Sánchez[1]1,2,a,c \\ 1 Instituto Nacional Materno Perinatal, Lima, Perú. \\ 2 Universidad Privada del Norte, Lima, Perú \\ a Médico cirujano; ${ }^{\mathrm{b}}$ especialista en Pediatría; ${ }^{\mathrm{c}}$ magíster en Razonamiento y Práctica Clínica.
}

\section{RESUMEN}

A pesar de las estrategias de prevención en la transmisión materno-infantil del virus de la inmunodeficiencia humana (VIH), continúan naciendo niños infectados. El objetivo del presente estudio fue describir el estado de los niños con VIH por transmisión materno-infantil. Entre 2012 y 2018, nacieron 84 niños con VIH en hospitales de Lima y Callao. De los 68 casos incluidos en este estudio, el 82\% de las madres no recibió tratamiento antirretroviral y el 25\% tuvo una prueba VIH negativa durante el embarazo. En cuanto a los niños, el 59\% fue diagnosticado de VIH después del año de edad, el $82 \%$ tiene actualmente carga viral menor de 1000 copias y el $87 \%$ tiene buen estado de salud. El diagnóstico y tratamiento temprano del niño con VIH le ofrece una mejor calidad de vida. Es necesario desarrollar estudios que identifiquen puntos críticos en la atención de la gestante para eliminar la transmisión vertical del VIH.

Palabras clave: Infecciones por VIH; Transmisión Vertical de Enfermedad Infecciosa; Recién Nacido; Salud del Niño (Fuente: DeCS BIREME).

\section{CHARACTERIZATION OF CHILDREN INFECTED WITH HIV DUE TO MOTHER-TO-CHILD TRANSMISSION IN HOSPITALS IN LIMA, PERU}

\begin{abstract}
Despite strategies to prevent mother-to-child transmission of the human immunodeficiency virus (HIV), infected children continue to be born. The aim of this study was to describe the status of children infected with HIV due to mother-to-child transmission. Between 2012 and 2018, 84 children were born with HIV in hospitals in Lima and Callao. Of the 68 cases included in this study, $82 \%$ of the mothers did not receive antiretroviral treatment and $25 \%$ had a negative HIV test during pregnancy. As for the children, $59 \%$ were diagnosed with HIV after one year of age, $82 \%$ had a viral load below 1,000 copies and $87 \%$ were considered healthy. Early diagnosis and treatment of the child with HIV is associated with better quality of life. It is necessary to carry out studies to identify the critical aspects of prenatal care in order to eliminate vertical HIV transmission.
\end{abstract}

Keywords: HIV Infections; Infectious Disease Transmission, Vertical; Newborn; Child Health (Source: MeSH NLM).

Citar como: Velásquez-Vásquez C, Espinola-Sánchez M. Caracterización de niños con VIH por transmisión materno-infantil atendidos en hospitales de Lima, Perú. Rev Peru Med Exp Salud Publica. 2020;37(4):694-99. doi: https:// doi.org/10.17843/rpmesp.2020.374.4816

Correspondencia: Carlos Velásquez Vásquez; Jr. Santa Rosa 941, Lima, Perú; carlos.carvel@gmail.com

Recibido: $19 / 09 / 2019$ Aprobado: 23/09/2020 En línea: 05/11/2020

\section{INTRODUCCIÓN}

La transmisión materno-infantil (TMI) del virus de la inmunodeficiencia humana (VIH) constituye la causa principal de transmisión del virus en menores de 15 años. Actualmente se conocen los mecanismos de la TMI, así como los protocolos de atención y los esquemas de tratamiento profiláctico para la gestante con VIH y el niño expuesto al virus ${ }^{(1,2)}$.

La atención en las embarazadas con VIH y la prevención de la TMI del virus se encuentran en la Norma Técnica 108-MINSA/DGSP «Prevención de la transmisión madre-niño del VIH y de la sífilis» del 2014. En ella se describen los procesos de diagnóstico precoz de la enfermedad en la gestante, el tratamiento antirretroviral, las medidas para la culminación del parto y la atención del niño expuesto ${ }^{(3)}$. Pese a lo anterior, todavía existe una incidencia de transmisión de, aproximadamente, $4 \%$ según la información obtenida de la Dirección de Prevención y Control del VIH, ITS y Hepatitis (DPVIH) y del Centro Nacional de Epidemiología, 
Prevención y Control de Enfermedades (CDC MINSA), lo cual está alejado de la meta propuesta por la Organización Mundial de la Salud que es menor del $2 \%{ }^{(4)}$.

Existen publicaciones nacionales que permiten medir el resultado de la aplicación de la norma nacional de prevención de la transmisión materno-infantil del VIH, así como el número reportado de niños infectados por el VIH en el país ${ }^{(5,6)}$. Por el contrario, es escasa la literatura existente dirigida al conocimiento del estado de salud de estos niños en los hospitales donde son atendidos mediante la NTS 102 MINSA/DGSP.V.01 «Norma Técnica de Salud para la Atención Integral y Tratamiento Antirretroviral de los Niños, Niñas y Adolescentes infectados por el Virus de la Inmunodeficiencia Humana (VIH)» del 2013, que describe los protocolos nacionales para la atención integral y tratamiento antirretroviral del niño con $\mathrm{VIH}^{(7)}$. El conocimiento de esta situación permitiría identificar brechas y plantear soluciones que garanticen el manejo adecuado del niño con VIH.

Por ello, el objetivo del presente estudio es conocer las características de los niños con VIH por TMI nacidos a partir del 2012 y que recibieron atención hasta el primer semestre del 2018 en los hospitales de Lima y Callao; así mismo, identificar puntos que pueden considerarse críticos para la transmisión del virus al niño.

\section{EL ESTUDIO}

Estudio descriptivo, donde se incluyeron a niños con VIH por TMI nacidos entre el 2012 y el primer semestre del 2018, seleccionados de entre todos los casos reportados a la DPVIH por los hospitales que realizan el seguimiento y atienden a los niños con VIH en Lima y Callao. Se excluyeron a los fallecidos, a quienes abandonaron el seguimiento, niños derivados a su centro de salud de origen fuera de Lima, los hospitalizados al momento del estudio, y a quienes no tenían historia clínica. Uno de los investigadores del estudio accedió a la información gracias al apoyo de los miembros del Comité de Expertos de Atención Integral del Niño con VIH (designados por Resolución Ministerial 1232-2003-SA/ DM), que es el grupo consultor de la DPVIH del Ministerio de Salud (MINSA).

El investigador del estudio usó una ficha de recolección de datos validada por el comité de expertos para recolectar información de las historias clínicas de los pacientes, de su ficha de seguimiento y de la entrevista con el médico tratante. En el estudio, se recogieron datos sobre la fecha de nacimiento y el lugar de origen del niño, la escolaridad, las fechas de diagnóstico de la infección (resultados de reacción en cadena de la polimerasa [PCR] y edad de diagnóstico), estado clínico del niño al momento del estudio, vía de transmisión del VIH, características de la TMI, profilaxis recibida ante la exposición al VIH, resultados iniciales y del último año de carga viral y de linfocitos CD4, y tratamiento antirretroviral

\section{MENSAJES CLAVE}

Motivación para realizar el estudio: Conocer el estado de los niños con virus de inmunodeficiencia humana $(\mathrm{VIH})$ por vía materna e identificar las causas de su transmisión a pesar de la aplicación del protocolo nacional.

Principales hallazgos: El diagnóstico del VIH en niños es tardío. El tratamiento antirretroviral mejora su calidad de vida. Los casos de niños con VIH se relacionan con madres sin diagnóstico y tratamiento antirretroviral oportuno.

Implicancias: Fortalecer el protocolo nacional en el diagnóstico y el seguimiento de la gestante con VIH y del niño expuesto, e identificar de manera oportuna las parejas discordantes disminuirán los nuevos casos de niños con VIH.

recibido (al inicio, de rescate y actual). Asimismo, se especificaron las ocurrencias de profilaxis en el escenario 3 según la Norma Técnica 108 MINSA/DGSP (las mujeres diagnosticadas por primera vez en el momento del parto reciben terapia antirretroviral en el momento; al niño se le administra profilaxis antirretroviral y se le suspende lactancia materna).

Los datos se procesaron usando el paquete estadístico IBM SPSS Statistics for Windows, versión 25 (IBM Corp., Armonk, N.Y., EUA). Las variables cualitativas se expresaron en medidas porcentuales, la carga viral al inicio del tratamiento se expresó en rangos. En todo el proceso de manejo de datos, se garantizó el anonimato de los participantes, mediante la codificación de su identidad y la protección de los datos a la que solo el personal del estudio tuvo acceso para fines del estudio.

El estudio fue realizado como parte de una intervención de vigilancia en salud pública de VIH por la DPVIH, incluida en su Plan Operativo Anual institucional del 2018.

\section{HALLAZGOS}

En el periodo de estudio se identificaron 84 niños con VIH, que reciben atención en seis hospitales de Lima (35 en el Instituto Nacional de salud del Niño, 19 en el Hospital Hipólito Unanue, 11 en el Hospital Nacional Cayetano Heredia, 10 en el Hospital Docente San Bartolomé, 7 en el Hospital Nacional Daniel Alcides Carrión del Callao, 2 en el Hospital Nacional Arzobispo Loayza). De los cuales, se incluyeron 68 niños con VIH por TMI, y se excluyeron cinco niños fallecidos, cuatro que abandonaron el seguimiento, tres que se derivaron a su centro de salud fuera de Lima, uno que fue hospitalizado, uno que no se infectó por TMI y uno cuya historia clínica no se identificó al momento del estudio.

De los 68 niños con VIH por TMI, la madre de 57 de ellos $(83,8 \%)$ no había recibido tratamiento durante el embarazo, y $7(10,3 \%)$ se consideraron escenario 3 . La edad de los niños en quienes se diagnosticó VIH fue entre 1 y 3 años (28 casos, 
Tabla 1. Características de la transmisión materno-infantil en niños con VIH

\begin{tabular}{|c|c|c|}
\hline Características & $\mathbf{n}$ & $\%$ \\
\hline \multicolumn{3}{|l|}{ Tratamiento antirretroviral de las madres } \\
\hline No recibió tratamiento ARV durante la gestación & 57 & 83,8 \\
\hline Escenario 3 & 7 & 10,3 \\
\hline No hay datos & 4 & 5,9 \\
\hline \multicolumn{3}{|l|}{ Tamizaje e identificación de VIH } \\
\hline No se encontraron registros de pruebas VIH durante el embarazo & 31 & 45,6 \\
\hline Prueba negativa para VIH durante la gestación & 17 & 25,0 \\
\hline Madre fue diagnosticada de VIH en un siguiente embarazo & 4 & 5,9 \\
\hline Padres fueron diagnosticados luego del nacimiento del niño & 5 & 7,3 \\
\hline Niños fueron diagnosticados en la etapa de lactante & 18 & 26,5 \\
\hline \multicolumn{3}{|l|}{ Lactancia materna } \\
\hline Sí recibieron & 45 & 66,2 \\
\hline No recibieron & 16 & 23,5 \\
\hline No se tiene información & 7 & 10,3 \\
\hline \multicolumn{3}{|l|}{ Edad de diagnóstico de VIH en niños } \\
\hline Menores de 6 meses & 16 & 23,5 \\
\hline De 6 meses a 12 meses & 5 & 7,4 \\
\hline Mayores de 1 año hasta 3 años & 28 & 41,2 \\
\hline Mayores de 3 años & 12 & 17,6 \\
\hline No se tiene información & 7 & 10,3 \\
\hline
\end{tabular}

VIH: virus de inmunodeficiencia humana, ARV: antirretroviral

\section{$41,2 \%$ ) y menos de seis meses (16 casos, 23,5\%) (Tabla 1).}

Los 68 niños están recibiendo tratamiento antirretroviral (TARV), 18 niños (26,5\%) presentaron rotación de esquema terapéutico, 59 niños $(86,8 \%)$ gozan de buen estado de salud, 5 (7,3\%) están desnutridos, 2 (2,9\%) tienen neumonía, 1 tiene tuberculosis y otro tiene síndrome de inmunodeficiencia adquirida (SIDA). Se encontró que 5,9\% de los niños son huérfanos y el total de niños en etapa escolar asisten regularmente a sus escuelas (Tabla 2).

Se obtuvieron datos de la última carga viral en el último año en 59 niños (a tres niños se les diagnosticó la infección en el último año antes del estudio y sus resultados estaban pendientes, y a 7 niños no se les encontró control de carga viral en el último año previo al estudio). En 34 niños $(57,6 \%)$ se presentó una carga viral indetectable en su último control, estos niños iniciaron tratamiento con cargas entre 30400 y 4006000 copias; 14 niños (23,7\%) presentaron menos de 1000 copias en su último control, estos iniciaron tratamiento con rangos de carga viral entre 7190 y 10400000 copias; 11 niños (18,7\%) presentaron más de 1000 copias en su último control de carga viral, ellos iniciaron tratamiento con carga virales en un rango de 52100 a 10000000 copias (Tabla 3).

\section{DISCUSIÓN}

El estudio permite observar que existe un alto porcentaje (59\%) de niños cuyo diagnóstico de VIH se realiza tardíamente, después del año de edad; a pesar de que la transmi- sión del virus se dio durante el embarazo o momento del parto. De niños en tratamiento antirretroviral, el $82 \%$ tiene carga viral menor a 1000 copias, lo que evidencia la eficacia del tratamiento recibido, y que el $87 \%$ goza de buena salud al

Tabla 2. Características del tratamiento y condiciones de niños con VIH

\begin{tabular}{lcc}
\hline Esquemas antirretrovirales & $\mathbf{n}$ & $\%$ \\
\hline Esquema de inicio & & \\
AZT + 3TC + LPV/r & 29 & 42,6 \\
AZT + 3TC + EFV & 12 & 17,6 \\
ABC + 3TC + LPV/r & 11 & 16,2 \\
AZT + 3TC + NVP & 5 & 7,3 \\
Otras combinaciones & 10 & 14,7 \\
No recibe tratamiento & 1 & 1,4 \\
Rotación de esquemas & & \\
Falta del TARV de inicio & 5 & 27,8 \\
Resistencia por genotipificación & 5 & 27,8 \\
Falta de adherencia & 5 & 27,8 \\
Anemia severa & 3 & 16,7 \\
Condiciones actuales de los niños con VIH & & \\
Buen estado de salud & 59 & 86,8 \\
Desnutrición & 5 & 7,3 \\
Neumonía & 2 & 2,9 \\
Tuberculosis & 1 & 1,5 \\
Estadio sida & 1 & 1,5 \\
Niños en orfandad & 4 & 5,9 \\
\hline
\end{tabular}

* El total de niños donde se rotó el tratamiento antirretroviral (ARV) fue de 18. AZT: zidovudina; TC: lamivudina; LPV/r: lopinavir reforzado con ritonavir; EFV: efavirenz; ABC: abacavir; NVP: nevirapina; VIH: virus de inmunodeficiencia humana; sida: síndrome de inmunodeficiencia adquirida; TARV: terapia antirretroviral. 
Tabla 3. Evolución de carga viral en niños con VIH que recibieron tratamiento antirretroviral $(\mathrm{n}=59)$

\begin{tabular}{llcc}
\hline Carga viral de control $\left(^{*}\right)$ & Rango de la carga viral al inicio del tratamiento & n & \% \\
\hline Indetectable & 30400 a 4006000 & 34 & 57,6 \\
Menores de 1000 copias & 7190 a 10400000 & 14 & 23,7 \\
Mayores de 1000 copias & 52100 a 10000000 & 11 & 18,7 \\
\hline
\end{tabular}

* Tres de los niños fueron diagnosticados en el último año antes del estudio y sus resultados estaban pendientes y a siete de los niños no se les encontró control de carga viral en el último año previo al estudio.

momento de culminar el estudio. En la búsqueda del probable momento crítico que pudo haber influido en la transmisión del virus de la madre al niño, se encontró que en el $82 \%$ de los casos, la madre no recibió tratamiento antirretroviral durante el embarazo, y el 25\% de las madres dio negativo en la prueba de VIH durante el embarazo, por lo que no recibieron ningún tratamiento para prevenir la TMI del virus.

Desde 1996, la prevención de la TMI del VIH formó parte de la política nacional de lucha contra el VIH/SIDA (Ley 26626), implementándose el tratamiento con zidovudina para la gestante con VIH, la cesárea y la suspensión de la lactancia materna. Desde esa fecha, se han generado una serie de mejoras en el programa que han ido de la mano con los avances del conocimiento en este campo ${ }^{(8,9,10)}$.

Estas estrategias han estado enfocadas a que las gestantes tengan más acceso a las pruebas del VIH, así como al TARV en la gestante con VIH y reducir la pérdida de seguimiento de los niños expuestos para optimizar la profilaxis antirretroviral, asegurar el diagnóstico precoz de la enfermedad en los niños a través de pruebas de PCR para VIH, y facilitar su inclusión en los servicios de atención pediátrica del VIH luego del diagnóstico ${ }^{(11)}$.

Los reportes presentados por el Centro Nacional de Epidemiología del MINSA permiten observar una tendencia favorable a la disminución de casos de VIH en niños, encontrándose una caída de 140 reportes en el 2000 a 40 casos o menos en los últimos cuatro años, no obstante, aún no se ha logrado llegar a la meta de eliminar la TMI del VIH, causa principal de la infección por el VIH en niños ${ }^{(6)}$.

Diferentes estudios nacionales tratan sobre la prevención de la TMI y su eficiencia, las investigaciones de Alarcón et al. ${ }^{(12)}$, y García et al. ${ }^{(13)}$ identifican algunos determinantes para la infección del VIH en la gestante, señalando que los riesgos se encontraban en el momento del embarazo y en las barreras para el acceso oportuno a los establecimientos de salud. Velásquez ${ }^{(5)}$ compara los protocolos de prevención de la TMI de VIH aplicados en el país y su efecto en la población atendida en el Instituto Nacional Materno Perinatal, y encontró una disminución de la TMI desde un $15 \%$, cuando se utilizaba solo zidovudina como profilaxis en la gestante con VIH, hasta un $4 \%$, cuando comenzó a utilizarse el tratamiento de gran actividad o TARGA. Por su parte, Huamán et al. ${ }^{(14)}$ identificaron factores que se constituyen en barreras para la aplicación de los protocolos de la prevención de TMI del VIH en poblaciones amazónicas. Asimismo, otros estudios miden la eficacia del diagnóstico con pruebas rápidas e intervenciones como la cesárea electiva ${ }^{(15,16)}$. A pesar de estos estudios realizados en el país, nos seguimos preguntando por qué siguen naciendo niños con VIH, lo cual es necesario responder para eliminar la TMI en el Perú.

Como se observa en el estudio, el diagnóstico de VIH en niños se realiza tardíamente (mayores de un año de edad), los que son tratados y seguidos en los hospitales de referencia. En muchos de los casos, no se tiene información respecto al embarazo materno y, por lo tanto, no se puede precisar el hecho crítico que pueda influir en la transmisión al niño. El diagnóstico y el tratamiento precoz en este grupo etario es un desafío debido a que existen niveles altos del virus en la sangre y los resultados de revisiones sistemáticas muestran que es preferible comenzar el TARV poco después del nacimiento en lugar de retrasarlo, debido a que los lactantes tienen menor probabilidad de morir o de enfermarse. Esto motiva a contar con sistemas de información que integren la atención materna y la del niño en todas las redes de salud, a fin de conocer el cumplimiento de los protocolos de diagnóstico del VIH en la gestante, el TARV, si fue positiva o las complicaciones durante este periodo; además permitiría la identificación precoz del niño con riesgo de infección o infectado y el inicio temprano del tratamiento ${ }^{(17)}$.

Un porcentaje importante de las madres fueron negativas a las pruebas del VIH en el embarazo y al momento del parto (25\%), pero posteriormente seroconvirtieron. En estos casos, el diagnóstico en el niño se hizo tardíamente, algunas veces en forma indirecta cuando los padres enfermaban y, otras, cuando el niño presentaba síntomas de inmunodepresión. Este hallazgo puede estar relacionado a problemas en la interpretación o calidad de las pruebas diagnósticas en los establecimientos de salud, o podría tratarse de parejas discordantes donde se desconoce el estado serológico de la pareja de una gestante seronegativa. En estos casos, la transmisión puede producirse después de las 24 semanas de gestación, en el parto o inmediatamente después del parto vaginal. Para disminuir esta posibilidad, debería implementarse una segunda prueba diagnóstica para el VIH en la gestante durante el último trimestre del embarazo y el ofrecimiento de la prueba de VIH a su pareja, esto permitiría la identificación y el manejo de parejas disconformes ${ }^{(18,19)}$.

Dentro del grupo de madres que no recibieron TARV, se encontró a las diagnosticadas oportunamente pero que 
abandonaron el tratamiento (falta de adherencia). Las causas de la falta de adherencia al tratamiento han sido estudiadas por Tejada ${ }^{(16)}$, quien considera que los cambios de rutina y otros factores complejos de tipo psicológico y social podrían explicar este abandono. Para enfrentar esta situación, es necesario fortalecer programas de seguimiento de la gestante con VIH, de tal manera que se asegure la adherencia al tratamiento ${ }^{(20)}$.

Otro hallazgo del estudio son las diferencias en el inicio del TARV en los niños, principalmente en aquellos que llegan de otras regiones del país. Si se considera que la NTS 102- MINSA/DGSP.V.01 dispone las combinaciones antirretrovirales para el inicio del TARV en niños a nivel nacional, es necesario corregir este problema mediante la capacitación continua descentralizada dirigida a los profesionales responsables de la atención de estos niños en el país ${ }^{(7)}$.

Las limitaciones de este estudio se deben a la inclusión de información retrospectiva a partir de registros médicos, lo cual podría implicar un sesgo de información. Además, se está determinando las características del niño con VIH por TMI cuyo seguimiento se encontraba disponible por los hospitales de Lima y Callao, pudiendo haber otros casos no reportados en el presente estudio. A pesar del ello, este estudio permite explorar y describir las condiciones de salud, de seguimiento y características de los niños con VIH, para identificar puntos críticos en la aplicación del protocolo de la prevención de la TMI, y generar intervenciones correctivas específicas.

En conclusión, la mayoría de los niños con VIH por TMI proceden de madres que no recibieron tratamiento antirretroviral durante la gestación, la mayoría de ellas no cuenta con registro de pruebas de VIH realizada durante la gesta- ción o presentó un resultado negativo. El mayor porcentaje de niños con VIH por TMI presentan un diagnóstico tardío, gozan por lo general de un buen estado de salud, mientras que en menor porcentaje se encontró desnutrición. Por otro lado, la mayor proporción de niños tiene una carga viral indetectable y continúan con su escolaridad de acuerdo con su edad. Se recomienda fortalecer las estrategias de prevención y de seguimiento del niño expuesto al VIH y continuar estudios en población infantil con VIH que permita identificar factores determinantes para su desarrollo y calidad de vida.

Agradecimientos: Un especial agradecimiento al grupo de expertos miembros de la Red de Manejo del Niño con VIH, consultores de la Dirección de Prevención y Control del VIH, ITS y Hepatitis del Ministerio de Salud, por el apoyo brindado en el acceso a la recolección de datos y ampliación de la información necesaria para el estudio en los diferentes hospitales: doctores Francisco Campos (Hospital Docente Madre Niño San Bartolomé), Yovana Mayor (Hospital Nacional Hipólito Unanue), Lenka Kolevic (Instituto Nacional de Salud del Niño), Guillermo Coronado y Lilia Guzmán (Hospital Daniel A. Carrión del Callao), Eduardo Verne (Hospital Nacional Cayetano Heredia), Debbie Miyasato (Hospital Nacional Arzobispo Loayza) y a la exdirectora de la DPVIH Mg. Patricia Segura.

Contribuciones de autoría: Todos los autores declaran haber participado en la concepción y diseño de la investigación, recolección de datos, análisis de datos, redacción del artículo, aprobación de la versión final y asumen la responsabilidad de su contenido.

Financiamiento: El estudio fue financiado por la Organización Panamericana de la Salud, Perú.

Conflictos de interés: Los autores no tienen ningún conflicto de interés que declarar.

\section{REFERENCIAS BIBLIOGRÁFICAS}

1. Lumaca A, Galli L, de Martino M, Chiappini E. Paediatric HIV-1 infection: updated strategies of prevention mother-to-child transmission. J Chemother. 2018;30(4):193-202. doi: 10.1080/1120009X.2018.1451030.

2. Fowler MG, Qin M, Fiscus SA, Currier JS, Flynn PM, Chipato T, et al. Benefits and Risks of Antiretroviral Therapy for Perinatal HIV Prevention. N Engl J Med. 2016;375(18):1726-1737. doi: 10.1056/NEJMoa1511691.

3. Ministerio de Salud. Norma Técnica de Salud para la prevención de la transmisión madre-niño del VIH y sífilis, 2014 [Internet]. Lima: Dirección General de Salud de las Personas, MINSA; 2014 [citado el 19 de mayo de 2019]. Disponible en: http://spij.minjus.gob.pe/Graficos/Peru/2014/ Agosto/19/RM-619-2014-MINSA.pdf.

4. Organización Panamericana de la Salud. Estrategia y plan de acción para la eliminación de la transmisión materno-infantil del VIH y de la sífilis congénita: Estrategia de monitoreo regional. 2014 [Internet]. Washington DC: OPS; 2014 [citado el 24 de mayo de 2019]. Disponible en: https:// iris.paho.org/bitstream/handle/10665.2/31365/9789275318126-spa. pdf? sequence $=1$ \&isAllowed $=y$.

5. Velásquez C. Resultados de la aplicación de tres guías nacionales para prevenir la transmisión vertical del VIH en el Instituto Nacional Materno Perinatal. Lima, Perú. Rev Peru Med Exp Salud Publica. 2011;28(3):492496. doi: 10.17843/rpmesp.2011.283.528.
6. Ministerio de Salud. Norma Técnica de Salud para la Atención Integral y Tratamiento Antirretroviral de los Niños, Niñas y Adolescentes infectados por el virus de inmunodeficiencia humana (VIH) [Internet]. Lima: Dirección General de Salud de las Personas; 2015 [citado el 28 de mayo de 2019]. Disponible en: http://bvs.minsa.gob.pe/local/MINSA/4858.pdf.

7. Ministerio de Salud. Situación epidemiológica del VIH-Sida en el Perú. Boletín mensual junio 2018 [Internet]. Lima: Centro Nacional de Epidemiología, Prevención y Control de Enfermedades, MINSA; 2018 [citado el 26 de mayo de 2019]. Disponible en http://www.dge.gob.pe/portal/ docs/vigilancia/vih/Boletin_2018/junio.pdf.

8. Ley referida al logro de objetivos del Plan Nacional CONTRASIDA, Ley No 26626 [Internet]. Diario Peruano; 18 de junio 1997 [citado 12 de agosto de 2019]. Disponible en: https://www.gob.pe/institucion/minsa/ normas-legales/256677-004-97-sa.

9. ACT UP Las Américas. Thalidomina y VIH/SIDA: Reporte del experimento ACTG-076 (AZT y embarazo: prevención de la transmisión de la madre al feto y/o infante) [Internet]. Cataluña: ACT UP Las Américas; 1994 [citado el 29 de mayo del 2019]. Disponible en: http://www.sidastudi. org/es/registro/2c9391e41fb402cc011fb43a09c117bd.

10. World Health Organization. Antiretroviral Drugs for Treating Pregnant Women and Preventing HIV Infection in Infants: Recommendations 
for a Public Health Approach: 2010 [Internet]. Geneva: World Health Organization; 2010 [citado el 28 de mayo de 2019]. Disponible en: https:// www.ncbi.nlm.nih.gov/books/NBK304945/.

11. Organización Panamericana de la Salud. Iniciativa Regional para la Eliminación de la Transmisión Maternoinfantil del VIH y de la Sífilis Congénita en América Latina y el Caribe: Estrategia de Monitoreo Regional [Internet]. Washington, D.C.: Latin American Center for Perinatology, Women and Reproductive Health (CLAP); 2010 [citado el 28 de mayo de 2019]. Disponible en: https://iris.paho.org/handle/10665.2/49406.

12. Alarcon JO, Johnson KM, Courtois B, Rodriguez C, Sanchez J, Watts $\mathrm{DM}$, et al. Determinants and prevalence of HIV infection in pregnant Peruvian women. AIDS. 2003;17(4):613-618. doi:10.1097/00002030200303070-00017.

13. García PJ, Nureña CR, Bayer AM, Cárcamo C, Lazo M, La Rosa S, et al. Nunca pensé que me iba a pasar a mí. VIH y vulnerabilidad de la mujer en el Perú: evidencias y recomendaciones para la acción [Internet]. Lima: Universidad Peruano Cayetano Heredia; 2013 [citado el 28 de mayo de 2019]. Disponible en: http://www2.congreso.gob.pe/sicr/cendocbib/ con4_uibd.nsf/C531EFAF98CB3BDF05257BBB005CB3D3/\$FILE/ UPCH-ONUSIDA-VIH-y-Vulnerabilidad-Mujer-Peru.pdf.

14. Huamán B, Gushiken A, Benites C, Quiroz F, Garcia-Fernández L. Prevención de la transmisión materno-infantil del VIH en gestantes y madres awajún y wampis de la región Amazonas en Perú. Rev Peru Med Exp Salud Pública. 2017;34(4):627-32. doi: 10.17843/rpmesp.2017.344.2725.
15. Melvin AJ, Alarcon J, Velasquez C, Rodriguez C, Piscoya J, Giraldo A, et al. Rapid HIV type 1 testing of women presenting in late pregnancy with unknown HIV status in Lima, Peru. AIDS Res Hum Retroviruses. 2004;20(10):1046-1052. doi: 10.1089/aid.2004.20.1046.

16. Tejada R, Alarcón J, Velásquez C, Gutiérrez C, Loarte C, Zunt J, et al. Factores asociados a la no adherencia al tratamiento antirretroviral de gran actividad durante la gestación, periparto y postparto en mujeres VIH positivas atendidas en el Instituto Nacional Materno Perinatal, Lima-Perú. Rev Peru Epidemiol. 2011;15(2):106-112.

17. Penazzato M, Prendergast AJ, Muhe LM, Tindyebwa D, Abrams E. Optimisation of antiretroviral therapy in HIV-infected children under 3 years of age. Cochrane Database of Systematic Reviews. 2014;(5):CD004772. doi: 10.1002/14651858.CD004772.pub4.

18. Taha TE, James MM, Hoover DR, Sun J, Laeyendecker O, Mullis CE, et al. Association of recent HIV infection and in-utero HIV-1 transmission. AIDS. 2011;25(11):1357-1364. doi: 10.1097/QAD.0b013e3283489d45.

19. Del Romero J, Baza MB, Río I, Jerónimo A, Vera M, Hernando V, et al. Natural conception in HIV-serodiscordant couples with the infected partner in suppressive antiretroviral therapy: A prospective cohort study. Medicine (Baltimore). 2016;95(30):e4398. doi: 10.1097/MD.0000000000004398.

20. Bailey H, Thorne C, Malyuta R, Townsend CL, Semenenko I, Cortina-Borja $\mathrm{M}$, et al. Adherence to antiretroviral therapy during pregnancy and the first year postpartum among HIV-positive women in Ukraine. BMC Public Health. 2014;14:993. doi: 10.1186/1471-2458-14-993. 